\title{
Clinical Observation on the Treatment of Hypertension by Combination of Modified Banxia Baizhu Tianma Decoction and Auricular
}

\section{Acupuncture}

\author{
Renjie Zhou, Liping Yang, Hongxing Zhang, Wangbo Cao* \\ Dezhou Zhang Hongxing Famous Senior TCM Physician Heritage Studio, Dezhou Hospital of Traditional Chinese \\ Medicine, Dezhou 253000, Shandong Province, China
}

\begin{abstract}
[Abstract] Objective: To observe the curative effect of Jiawei Banxia Baizhu Tianma Decoction (BBTD) in combination with auricular acupuncture in the treatment of primary hypertension with phlegm-dampness syndrome. Methods: 120 patients with hypertension were randomly divided into a pure Western medicine treatment control group (referred to as the control group) and an integrated traditional Chinese and Western medicine treatment group (referred to as the treatment group), with 60 cases in each group. The control group was given nifedipine sustained-release tablets orally; the treatment group: nifedipine sustained-release tablets were given on the basis of modified BBTD combined with auricular acupuncture. The treatment course duration of the two groups was 4 weeks. Before and after treatment, the two groups were evaluated for treatment efficacy and quality of life. Results: There is statistically significant difference in blood pressure lowering between the treatment group and the control group $(\mathrm{P}<0.05)$; the treatment group was better than the control group in symptoms improvement of the patients $(\mathrm{P}<0.05)$; in terms of the improvement in quality of life, the treatment group was significantly better than the control group ( $\mathrm{P}<0.05, \mathrm{P}<0.01$ ). Conclusion: Modified BBTD combined with auricular acupuncture method can reduce blood pressure and improve clinical symptoms in patients with primary hypertension with phlegm-dampness syndrome, and significantly improve the quality of life of patients.
\end{abstract}

Key words: Vertigo; Banxia Baizhu Tianma Decoction; Phlegm dampness; Auricular acupuncture; Quality of life

Publication date: May, 2021; Publication online: 31 May, 2021

*Corresponding author: Wangbo Cao, cao800503@163.com

\section{Introduction}

Hypertension is one of the most common clinical diseases in China. It often causes elevated blood pressure, increased blood vessel wall stiffness, aggravated arteriosclerosis, and damages to target organs such as heart, brain, and kidney, which seriously threaten people's health. According to "China Cardiovascular Disease Report 2018", there are 290 million people suffering from cardiovascular disease in China, including 245 million patients with hypertension, and the prevalence rate is increasing year by year ${ }^{[1]}$. The prevalence of adult hypertension in China was $27.9 \%$, which increases with age. The diagnostic rate of hypertension was $51.6 \%$, and the treatment control rate was $37.5 \%$. Traditional antihypertensive drugs focus on reducing peripheral vascular resistance and have limited effects on improving patients' symptoms and alleviating negative emotions. Therefore, we seek to treat more comprehensively from the perspective of Chinese medicine.

\section{Information and methods}

\subsection{Case sources}

The cases in this study were all from the inpatients at the Department of Cardiology, Dezhou Hospital of Traditional 
Chinese Medicine from May 2019 to May 2020, with a total of 120 cases. The first diagnosis was the diagnosis of traditional Chinese medicine: vertigo (phlegm-dampness syndrome); the diagnosis of Western medicine: essential hypertension (level I, II). 120 patients were randomly divided into the treatment group and the control group at a ratio of 1:1. After statistical analysis, there was no statistical difference between the two groups in terms of gender, age, course of disease, and blood pressure $(\mathrm{P}>0.05)$.

\subsection{Diagnostic criteria}

The diagnostic criteria of TCM refer to the criteria established in the "Guiding Principles of Clinical Research on New Chinese Medicines", that is, the TCM diagnosis of hypertension is the syndrome of excessive phlegm-dampness, with 1 primary syndrome + at least 2 secondary syndromes, the diagnosis is established; Western medicine diagnostic standards refer to the standards stated in the "Guidelines for Prevention and Treatment of Hypertension in China 2010"[2, 3].

\subsection{Inclusion criteria}

(1) Participated voluntarily and cooperated with physicians, and signed a written informed consent; (2) Meet the diagnostic criteria for phlegm and dampness under the "Guidelines for the Prevention and Treatment of Hypertension in China (2010 Revised Edition)"; (3) Age over 18, gender not restricted ${ }^{[3]}$.

\subsection{Exclusion criteria}

Those with one of the following conditions were excluded:

(1) Other diseases that may cause increased cardiac output and systolic blood pressure, such as severe anemia, hyperthyroidism, aortic insufficiency, patent ductus arteriosus, etc.; (2) Unable to finish the complete collection of syndromes and related medical history data, and/or unwilling or unable to comply with the researchers to complete the hypertension epidemiological questionnaire.

\subsection{Removal criteria}

After inclusion, cases that were found to not meet the inclusion criteria and with questionnaire blank content $>$ $20 \%$ was removed from the study.

\subsection{Experimental method}

All patients had a low-salt and low-fat diet, and abstained from smoking and alcohol.

Control group: Oral Degaoning (Nifedipine Sustained Release Tablets, Chinese Drug Standard Serial No.: H10920005, Dezhou Deyao Pharmaceutical Co., Ltd.) (10mg/tablet). Administered for 4 weeks.
Treatment group: The treatment group received the above-mentioned control group drug orally with the addition of Banxia Baizhu Tianma Decoction (BBTD): Banxia (pinellia) 9g, Baizhu (atractylodes) 20g, Tianma (gastrodia) $30 \mathrm{~g}$, poria $15 \mathrm{~g}$, tangerine $15 \mathrm{~g}$, licorice $6 \mathrm{~g}$, eucommia $12 \mathrm{~g}$, Alisma orientalis $20 \mathrm{~g}$, and tangerine peel $12 \mathrm{~g}$. $400 \mathrm{ml}$ of the juice from the decoction was taken daily in $200 \mathrm{ml}$ servings, once in the morning and once in the evening; duration time 4 weeks. Auricular acupuncture points: liver, spleen, stomach, hypertension, adrenal gland, Shenmen. $75 \%$ alcohol cotton ball was used to disinfect the part of the acupuncture points, then the Wangbuliuxing seeds were sticked to the acupuncture points. The thumb and index finger were used to press the acupuncture points lightly, from light to heavy. Each acupoint was pressed for an average of 3 minutes, three times a day, and the dressing was changed every day, alternating the ears. The total duration of treatment is 4 weeks.

\subsection{Observation indicators}

\subsubsection{Blood pressure}

Blood pressure was measured at fixed time every day.

\subsubsection{Scoring symptoms}

According to the "Guiding Principles for Clinical Research of New Chinese Medicines" published by China Medical Science and Technology Press in 2002, the quantification table of hypertension symptom grading and quantification was used to score the symptoms. Main symptoms: dizziness, headache, head-wrapped, chest tightness, vomiting, sputum and salivation; secondary symptoms: palpitations, insomnia, light mouth, lack of appetite, fat tongue, greasy coating, slippery pulse, indicated by different scoring methods according to the major and minor symptoms and their degrees. The main symptoms are counted as $0,2,4$, and 6 according to the difference between normal, mild, moderate, and severe, and the secondary symptoms are counted as 0,1 , 2 , and 3 according to the difference between normal, mild, moderate, and severe. Scoring was made before and after treatment, and the changes in scoring before and after treatment was observed.

\subsubsection{Evaluation of quality of life}

The patients' quality of life was evaluated according to the World Health Organization Quality of Life Scale.

\subsection{Treatment efficacy assessment criteria}

\subsubsection{Assessment criteria for antihypertensive efficacy}

Evaluate the antihypertensive efficacy was assessed based on the "Guidelines for Prevention and Treatment of 
Hypertension in China"[2]. Significantly effective: Diastolic blood pressure (DBP) decreased by $\geq 20 \mathrm{mmHg}$, or DBP decreased by $\geq 10 \mathrm{mmHg}$ and reached the normal value; Effective: $10 \mathrm{mmHg}<$ DBP decreased by $<20 \mathrm{mmHg}$, or DBP decreased by $<10 \mathrm{mmHg}$ and reached the normal value, or systolic blood pressure (SBP) decreased by $\geq 30$ $\mathrm{mmHg}$; Invalid: not reaching any of the above levels.

2.8.2 The curative effects of traditional Chinese medicine on symptoms

The curative effects of traditional Chinese medicine on symptoms refer to the quantification table of hypertension symptom classification in the "Guiding Principles for Clinical Research of New Chinese Medicines" published by China Medical Science and Technology Press in 2002 [2].

\subsubsection{Quality of life assessment standard}

Quality of life assessment standard Refers to the Quality of
Life Scale for Patients with Hypertension (QOL Assessment Scale).

\subsection{Statistical processing}

All statistical results were calculated using the SPSS Statistics 19.0 software. The measurement data were expressed in the form of mean \pm standard deviation $\left(\bar{X}_{ \pm S}\right)$, and the t-test was applied after the homogeneity of variance test; $\mathrm{X}^{2}$ test was applied to the count data, and the rank sum test was applied to the rank data.

\section{Statistical results}

\subsection{Antihypertensive efficacy}

The total effective rate of the experimental group was $88.3 \%$, and that of the control group was $91.6 \%$. There was no statistical difference between the groups $(P>0.05)$. See Table 1.

Table 1. Comparison on antihypertensive efficacy between control and experimental group

\begin{tabular}{cccccc}
\hline Group & Cases & Significantly Effective & Effective & Invalid & Total Effective Rate (\%) \\
\hline Control & 60 & 22 & 33 & 7 & 88.3 \\
Experimental & 60 & 28 & 30 & 5 & 91.6 \\
\hline
\end{tabular}

\subsection{Symptom relief score}

significantly improved in symptoms, which is statistically

The hypertensive patients in the experimental group different from the control group $(\mathrm{P}<0.05)$. See Table 2.

Table 2. Comparison on symptom relief score between control and experimental group before and after treatment

\begin{tabular}{cccc}
\hline Group & Cases & Before Treatment & After Treatment \\
\hline Control & 60 & $15.38 \pm 3.12$ & $11.68 \pm 2.84^{*} \#$ \\
Experimental & 60 & $15.31 \pm 2.58$ & $13.72 \pm 2.53$ \\
\hline
\end{tabular}

Note: Compared to before treatment, ${ }^{*} P<0.05$; compared to control group, ${ }^{\#} P<0.05$.

\subsection{Assessment of quality of life}

After treatment, the experimental group had statistical differences in physiological functions, physical functions, and psychological emotions compared to before treatment
$(\mathrm{P}<0.05, \quad \mathrm{P}<0.01)$. In terms of physical functions and psychological emotions, there were significant statistical differences between the experimental group and the control group after treatment $(\mathrm{P}<0.01)$. See Table 3 .

Table 3. Comparison on assessment of quality of life between control and experimental group before and after treatment

\begin{tabular}{cccccc}
\hline \multirow{2}{*}{ Group } & \multicolumn{2}{c}{ Experimental $\boldsymbol{n}=\mathbf{6 0}$} & & \multicolumn{2}{c}{ Control $\boldsymbol{n = 6 0}$} \\
\cline { 2 - 3 } & Before Treatment & After Treatment & & Before Treatment & After Treatment \\
\hline Physiological Functions & $27.57 \pm 3.78$ & $32.36 \pm 3.22^{* *} \# \#$ & & $28.14 \pm 3.91$ & $29.41 \pm 2.36^{*}$
\end{tabular}




\begin{tabular}{ccccc} 
Physical Functions & $22.98 \pm 1.78$ & $23.48 \pm 1.69^{*}$ & $23.04 \pm 1.72$ & $23.35 \pm 1.81$ \\
Daily Life & $29.68 \pm 3.22$ & $30.22 \pm 3.28$ & $29.97 \pm 3.26$ & $30.31 \pm 3.21$ \\
Psychological Emotions & $18.47 \pm 3.22$ & $20.67 \pm 2.78^{* *} \# \#$ & $18.63 \pm 3.11$ & $18.89 \pm 3.03$ \\
\hline
\end{tabular}

Note: Compared to before treatment, ${ }^{*} P<0.05,{ }^{*}{ }^{*} P<0.01$; compared to control group, ${ }^{\#} P<0.05,{ }^{\# \#} P<0.01$.

\section{Discussion}

Hypertension belongs to the category of vertigo in Chinese medicine, and its clinical symptoms are dizziness and headache. Banxia Baizhu Tianma Decoction (BBTD) was derived from the "Medical Enlightenment" by Cheng Guopeng in Qing Dynasty. The book wrote that, "Dizziness (xuan) means vision turning dark; fainting (yun) means vertigo, which is also called yanhua (giddiness) in ancient times..... For those who have damp phlegm congestion, as written in the book: "Dizziness and vertigo cannot be cured without Tianma (gastrodia) and Banxia (pinellia)", BBTD should be the main medicine..... Banxia Baizhu Tianma Decoction: One qian and five fen of Banxia, one qian of Tianma, poria, tangerine each, three qian of Baizhu, five qian of licorice, one piece of ginger, two jujubes, made into decoction and drink". The book further mentions that this prescription can also be used to treat headaches, that is, "for people with phlegm induced headaches, phlegm in the chest and lungs, and dizziness when moving around, BBTD is the main medicine". It is generally believed that the liver 'wind' and phlegm are the most important pathology of this syndrome. This prescription works to calm the liver, eliminate 'wind', resolve phlegm and relieve vomiting. In the prescription, Banxia is used to dry dampness and resolve phlegm, reduce adverse effects and stop vomiting; Tianma can calm the liver, eliminate 'wind' and stop dizziness; the two are used together to form the 'lord' medicine. With Baizhu as the 'minister', it invigorates the spleen and dries dampness, and is compatible with Banxia and Tianma, eliminating dampness and phlegm to stop dizziness. It is supplemented with poria to invigorate the spleen and seep dampness, and combined with Baizhu to treat the root cause of phlegm. Many modern doctors apply this prescription to the treatment of hypertension. Mr. Yue Meizhong ${ }^{[4]}$ proposed that this prescription can be used for weak spleen, phlegm and dampness resistance, upside-down of stomach $q i$, and severe dizziness, where blood pressure fluctuates greatly like a rowing boat. He believes that this prescription can not only be used to treat weak spleen and upside-down of phlegm-qi, but also adjust the overall body function, especially for people with large fluctuations in blood pressure. It can also be used for people who suffer from headache and sleepiness after eating and low blood pressure. People with high blood pressure due to weakness of the spleen and stomach, headache and fatigue, can regulate the circulation of $q i$ and blood, make $q i$ and blood vigorous, and regulate overall functions. Therefore, Mr. Yue Meizhong believes that this prescription has a two-way effect in regulating blood pressure. In clinical pharmacological studies, BBTD can adjust the serum NO concentration and endothelin-1 level, increase the serum NO concentration, and reduce the levels of serum angiotensin II and endothelin-1, thereby improving vascular endothelial function and lowering blood pressure by regulating the endocrine system ${ }^{[5]}$. Sun Fujun et $a l^{[6]}$ observed experimentally the effect of BBTD on the relevant indicators of hyperlipidemia model rats and found that BBTD had a significant intervening effect on the blood lipid level of hyperlipidemia model rats. It also lowers serum cholesterol, triglycerides, low density lipoprotein and apolipoprotein B, and increases the effect of high density lipoprotein. Jiang Jiaye $^{[7]}$ explored the changes in the RAS system of hypertrophic myocardium in spontaneously hypertensive rats, and observed the effects of BBTD in different periods on the hemodynamics and the mRNA of various factors in RAS. The study found that changes in RAS occurred in the hypertrophic myocardium of SHR, which may be one of the molecular mechanisms leading to hypertension and left ventricular hypertrophy. Application of BBTD can improve hemodynamic indicators, regulate RAS and lower arterial pressure. Jiang Lingyu et $a l^{[8]}$ used proteomics as the starting point to explore the antihypertensive mechanism of BBTD, separated samples by two-dimensional electrophoresis and staining with silver nitrate, then screened different protein spots for mass spectrometry analysis and protein identification. The difference in protein expressions was further identified by mass spectrometry. Among the 19 proteins identified, the model group was different from the normal group, while the BBTD could recover 11 protein 
spots, of which 5 expressions were up-regulated and 6 expressions were down-regulated, suggesting that these differential proteins may be the target of the antihypertensive effect of BBTD, and these proteins are related to cell morphology, oxidative stress and energy metabolism. Therefore, the molecular mechanisms of BBTD antihypertensive therapy may lie in controlling the proliferation of tubulin and inhibiting neuronal cell apoptosis; enhancing the production of ATP and increasing the energy supply of neurons; improving internal plasma reticulum stress, maintaining the basic structure of neurons, to ensure the signaling function is normal and maintain the cytoskeleton and morphology.

Traditional Chinese medicine believes that the ears have internal connections with the internal organs through the fourteen meridian collaterals; and it can also reach the body hair through the external collaterals of the fourteen meridians, which leads to the functions of activating the meridians, regulating $q i$ and blood, and regulating internal organs. Auricular acupuncture is mainly a treatment method for pressing and massaging the acupoints of the patient's ears. At the same time, it is a new type of treatment mode combined with targeted nursing methods, which can effectively relieve the symptoms of dizziness and headache. It also has a certain therapeutic effect on the control of blood pressure. The Wangbuliuxing seeds are antihypertensive medicine with good effects on promoting blood circulation and removing blood stasis. Long-term rubbing and pressing can adjust the body's essence, $q i$ and blood, dredge the veins, improve the function of the organs, promote $q i$ and blood circulation, and finally achieve the goal of calming the liver and lowering blood pressure ${ }^{[9]}$. As one of the external treatments for hypertension, auricular acupuncture plays an important role in reducing blood pressure and regulating internal organs. In this method, the liver acupoints can sooth the liver and regulate $q i$, clear the liver and purge 'fire', the spleen acupoints and the stomach acupoints can regulate Zhongzhou, dissipate phlegm and dampness, the adrenal acupoints can nourish kidney yin, hypertension acupoints are Ashi acupoints that can reduce blood pressure, and Shenmen acupoints regulate the heart and veins. Together they enhance the spleen and remove dampness, sooth the liver and regulate $q i$.

This study shows that BBTD combined with auricular acupuncture can effectively reduce the blood pressure of hypertension patients, improve clinical symptoms, improve the quality of life, and relieve negative emotions. Based on the fundamental theories of traditional Chinese medicine, the advantages of the Chinese medicine are brought into play, which not only enables the patient's objective indicators to be controlled, but also improves subjective feelings. Of course, the sample size of this trial is small, and further large-sample RCT experiments are needed in clinical practice to standardize the advantages of traditional Chinese medicine in lowering blood pressure.

\section{References}

[1] Hu SS, Gao RL, Liu LS, et al. China Cardiovascular Disease Report 2018 (Summary) $[\mathrm{J}]$. Chinese Circulation Journal, 2019, 34 (3): 209 - 220.

[2] Zheng XY. Guidelines for Clinical Research of New Chinese Medicines[M]. Beijing: China Medical Science Press, 2002: 73 .

[3] Liu LS. 2010 Chinese guidelines for the management of hypertension $[\mathrm{J}]$. Chinese Journal of Hypertension,2011, 19( 8): 701.

[4] Zhang JC. Diagnosis and Treatment of Heart Disease [N]. China News of Traditional Chinese Medicine,2012-05-30(5)

[5] Li F, Li X. Effect of the Combination of Banxia Baizhu Tianma Decoction and Xuefu Zhuyu Decoction on Endothelial Function and for Treating Hypertension [J].Guangdong Medical Journal,2014,35(6):936-937

[6] Sun FJ, Huang WK, Li XJ, Wang JF, Chen HH, Wang CF, Wang QL. Study on treatment of hyperlipidemia by Banxia Baizhu Tianma decoction [J]. Chinese Journal of Experimental Traditional Medical Formulae,2010,09:169-172.

[7] Jiang JY, Wang XZ, Luo SS, Wang X, Bian K, Ke Y. Effect of Banxia Baizhu Tianma decoction on the left ventricular hypertrophy of hypertrophied myocardium in spontaneously hypertensive rat $[\mathrm{J}]$. Chinese Journal of Integrated Traditional and Western Medicine,2010,10:1061-1066.

[8] Jiang LY, Jiang YH, Yang CH, et al. Effect of Banxia Baizhu Tianma decoction on proteomics of hypothalamus in rat model of hypertension with excessive phlegm and dampness [J]. Journal of Traditional Chinese Medicine,2017,58(13):1139-1144.

[9] Liu QH. Clinical research progress of traditional Chinese medicine in treating hypertension [J]. Journal 
(1) :98-101. 